Document downloaded from:

http://hdl.handle.net/10251/120534

This paper must be cited as:

De Oña, J.; López-Maldonado, G.; Abellán, J. (2013). Extracting decision rules from police accident reports through decision trees. Accident Analysis \& Prevention. 50:1151-1160. https://doi.org/10.1016/j.aap.2012.09.006

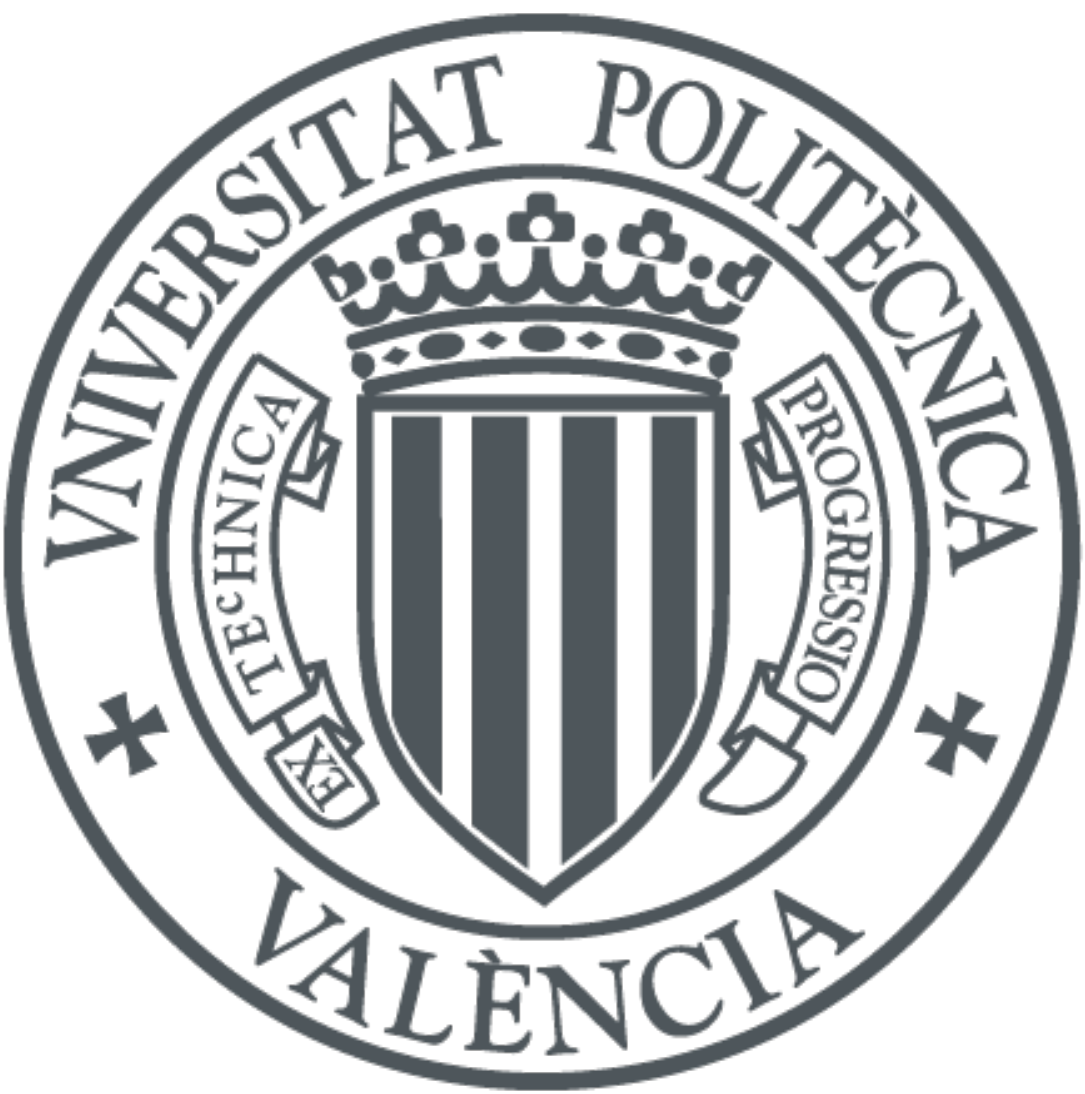

The final publication is available at

http://dx.doi.org/10.1016/j.aap.2012.09.006

Copyright Elsevier

Additional Information 


\title{
Extracting decision rules from police accident reports through decision trees
}

\author{
Juan de Oña ${ }^{\mathrm{a}, *}$, Griselda López ${ }^{\mathrm{a}}$ and Joaquín Abellán ${ }^{\mathrm{b}}$ \\ a TRYSE Research Group. Department of Civil Engineering, University of Granada, ETSI \\ Caminos, Canales y Puertos, c/ Severo Ochoa, s/n, 18071 Granada (Spain) \\ ${ }^{\mathrm{b}}$ Department of Computer Science \& Artificial Intelligence, ETSI Informática, c/Periodista \\ Daniel Saucedo Aranda, s/n, 18071 Granada (Spain)
}

* Corresponding author. Phone: +34 9582499 79, jdona@ugr.es

\begin{abstract}
Given the current number of road accidents, the aim of many road safety analysts is to identify the main factors that contribute to crash severity. To pinpoint those factors, this paper shows an application that applies some of the methods most commonly used to build Decision Trees (DTs), which have not been applied to the Road Safety field before. An analysis of accidents on rural highways in the province of Granada (Spain) between 2003 and 2009 (both inclusive) showed that the methods used to build DTs serve our purpose and may even be complementary. Applying these methods has enabled potentially useful decision rules to be extracted that could be used by road safety analysts. For instance, some of the rules may indicate that women, contrary to men, increase their risk of severity under bad lighting conditions. The rules could be used in road safety campaigns to mitigate specific problems. This would enable managers to implement priority actions based on a classification of accidents by types (depending on their severity). However, the primary importance of this proposal is that other data bases not used here (i.e. other infrastructure, roads and countries) could be used to identify unconventional problems in a manner easy for road safety managers to understand, as decision rules.
\end{abstract}

Keywords: traffic accident; severity; decision trees; CART; C4.5; decision rules

\section{Introduction}

Traffic accidents are considered a major public health problem worldwide, claiming 1.27 million annual deaths and between 20 and 50 million injuries (WHO, 2009). Therefore, the aim of many studies to date has been to understand and identify the main factors that have an impact on road accident severity. Regression-type generalized linear models, Logit models and Probit models have been the techniques most commonly used to conduct such analyses (Kashani and Mohaymany, 2011; Savolainen et al., 2011; Mujalli and De Oña, in press). However most of them have their own model assumptions and pre-defined underlying relationships between dependent and independent variables (Chang and Wang, 2006).

Recently, data mining (DM) techniques have been used to study crash-injury severities by different researchers (Kuhnert et al. 2000; Sohn and Shin, 2001; Chang and Wang, 2006; Kashani and Mohaymany, 2011; Kashani et al., 2011; Pakgohar et al., 2010). The term Decision Trees (DTs) encompasses a series of techniques for extracting processable knowledge, implicit in data bases, which is based on artificial intelligence and statistical analysis. One part of the DM could be defined as the non trivial process of identifying valid, novel, potentially useful and ultimately understandable pattern in data (Fayyad et al., 1996). These techniques are aimed at extracting knowledge from large amounts of data previously unknown and indistinguishable. DT techniques are particularly appropriate for studying crashes because they are non-parametric techniques that do not require prior probabilistic knowledge on the study phenomena. 
Furthermore, they consider conditional interactions among input data (Montella et al., 2011). Other advantages of DTs compared to other methods with similar aims include the extraction of decision rules of the "if-then" type (Kashani et al., 2011), and that they can be used to discover behaviours that occur within a specified set of data. Moreover, conclusions on behavior can be drawn from the structure of DTs to understand the events leading up to a crash and identify the variables that determine how serious an accident will be.

There are many algorithms that can be used to build DTs, but CART (Classification and Regression Trees) developed by Breiman et al. in 1984 is the once most commonly used to analyze crash severity. Authors such as Kuhnert et al. (2000) compared the results obtained with CART, multivariate adaptive regression splines (MARS) and logistic regression in the analysis of an epidemiological case-control study of injuries resulting from motor vehicle accidents. The findings indicated that non-parametric techniques such as CART and MARS can provide more informative and attractive models whose individual components can be displayed graphically. Chang and Wang (2006) studied the relationships between crash severity with characteristics related to drivers and vehicles, as well as variables related to roads, road accidents and the environment characteristics. Pakgohar et al. (2010) used CART and Multinomial Logistic Regression to study the role played by drivers' characteristics in the resulting crash severity. They found that the CART method provided more precise results, which were also simpler and easier to interpret. Kashani et al. (2011) studied the key factors that affect the injury severity of drivers involved in crashes on two-lane two-way rural roads. Subsequently, Kashani and Mohaymany (2011) used CART to identify the main factors that affect the injury severity of vehicle occupants involved in crashes on those roads.

However, CART always yields binary trees, which sometimes cannot be summarized as efficiently for interpretation and/or presentation (Breiman et al., 1984). In the case of road accidents, they may not be very practical when it comes to analyzing the impact of a specific category of variable in crash severity. Liu (2009) mentions the existence of other popular algorithms for building DTs, such as C4.5. He does not apply it, however, because a binary DT is sufficient to develop his work.

Other simple algorithms, such as ID3 (Quinlan, 1986) and C4.5 (Quinlan, 1993), have been widely used in the literature of DM for building DTs, and do not involve the binary restriction. Therefore, this study proposes to make a comparison between the various methods, and to use CART and other methods to identify the main factors that affect crash severity. Then we extract certain decision or association rules (Agrawal et al., 1993) from the methods that give the best results. We show that the methods used complement our objective. Finally, our results could be used for the predictive purposes pursued by road safety analysts.

This paper is organized as follows: Section 2 gives an introduction to procedures for building DTs, focusing on the ones used in this study. It also describes the parameters used to assess the various methods, the procedure for extracting rules and the main features of the study data. Section 3 presents the results and a discussion on them. Finally, the last section presents the conclusions.

\section{Materials and methods}

\subsection{Decision Trees}

A DT is a predictive model which can be used to represent both classifiers and regression models. DTs are popular due to their simplicity and transparency; moreover, they are usually presented graphically as hierarchical structures, which make them easy to interpret. 
A DT is a simple structure that can be used as a classifier. Within a DT, each node represents an attribute variable ${ }^{1} X$ and, each branch represents one of the states of this variable. Normally, a terminal node or leaf, specifies the expected value of the class variable or variable in study $\mathrm{C}$, depending on the information contained in the training data set, i.e. the set used to build the model. The set of data used to check the model is called test set. When we obtain a new instance or case of the test data set, we can make a decision or prediction about the state of the variable class following the path to the tree from the root node to a leaf node, using the sample values and the tree structure. Subsequently, the model obtained can be used to classify new examples (cases whose classes are not known a priori), to detect patterns, or simply to gain a better understanding of the phenomenon being analyzed.

DTs are built recursively, following a descending strategy, starting with the full data set (made by the root node). Using specific split criteria, the full set of data is then split into even smaller subsets. Each subset is split recursively until all of them are pure (when the cases in each subset are all of the same class) or their "purity" cannot be increased. That is how the tree's terminal nodes are formed, which are obtained according to the answer values of the class variable.

The main difference between DTs building procedures lies in the splitting criteria. The most commonly applied splitting criteria in simple algorithms are the Gini Index (which measures the degree of purity), used in the CART system (Breiman et al., 1984); Information Gain, used in the ID3 algorithm (Quinlan, 1986); and the Information Gain Ratio, used in the C4.5 algorithm (Quinlan, 1993). ID3 and C4.5 are based on the entropy, which measures the degree of confusion (the greater the confusion, less information). The procedures also differ in the strategies they use after building a tree, in the process known as pruning. This is when the model obtained is simplified and adjusted more closely to the data set used to build it.

\subsection{Methods for building Decision Trees}

CART: Depending on the nature of the dependent variable, a classification tree (case discrete) or a regression tree (case continuous) will be built. The CART model generates binary trees by using impurity as a measure to split the Gini Index of diversity (which is a measure of the diversity of classes in a tree node being used). For a variable $\mathrm{C}$, it is defined as:

$$
\operatorname{gini}(C)=1-\Sigma_{j} p^{2}\left(C=c_{j}\right) .
$$

In this way, we can define the split criterion based on the Gini Index as:

$$
\mathrm{GI} x(C, X)=\operatorname{gini}(C \mid X)-\operatorname{gini}(X),
$$

where $\operatorname{gini}(C \mid X)=\sum_{t} p\left(x_{t}\right) \operatorname{gini}\left(C \mid X=x_{t}\right)$ and $X$ another known variable.

Thus, the best split is the one that minimizes $\mathrm{GI} x\left(C_{y} X\right)$. With this procedure, the maximal tree that overfits the data is created. To decrease its complexity, the tree is pruned using a costcomplexity measure that combines the precision criteria as opposed to complexity in the number of nodes and processing speed, searching for the tree that obtains the lowest value for this parameter. A more detailed description of the CART method can be found in Breiman et al. (1984).

ID3: Builds a tree in a manner similar to the CART method but without the binary restriction. It can only be used with discrete variables, does not allow pruning and the function used to measure impurity is the Shannon's entropy (Shannon, 1948), which is an information-based uncertainty measure.

\footnotetext{
${ }^{1}$ Also called feature or predictor variable
} 
The ID3 algorithm uses the Information Gain criterion to choose which attribute goes into a decision node. Information Gain could be defined as a difference of entropies in the current node, considering the information that an attribute variable gives us about the class variable. This split criterion can therefore be defined on an attribute variable $\mathrm{X}$, given the class variable $\mathrm{C}$, as follow:

$$
\text { Information Gain }(C, X)=I G(C, X)=H(C)-H(C \mid X)
$$

Where $H(C)$ is the entropy of $\mathrm{C}, H(C)=-\Sigma_{j} p\left(c_{j}\right) \log p\left(c_{j}\right)$, with $p\left(c_{j}\right)=p\left(C=c_{j}\right)$, the probability of each value of the variable class estimated in the training data set. In the same way, $H(C \mid X)=-\Sigma_{t} \Sigma_{j} p\left(c_{j} \mid x_{t}\right) \log p\left(c_{j} \mid x_{t}\right)$, where $x_{t}, \mathrm{t}=1, \ldots,|\mathrm{X}|$, is each possible state of $\mathrm{X}$ and $c_{j}, \mathrm{j}=1, \ldots, \mathrm{k}$ each possible state of $\mathrm{C}$.

Notice that the Information Gain criterion has implicit preference for splitting nominal attributes with lots of values. Therefore, it produces trees that discard the remaining attributes prematurely because they soon come to branches that have only a few cases. A more detailed description of the ID3 algorithm can be found in Quinlan (1986).

C4.5: In order to improve the ID3 algorithm, Quinlan (1993) introduces the C4.5 algorithm, where the Information Gain split criterion is replaced by an Information Gain Ratio criterion which penalizes variables with many states. Moreover, this model makes it possible to deal with continuous attributes and missing values, and to carry out a post-pruning process. The algorithm incorporates classification tree pruning once a tree has been induced, by applying a hypothesis test on whether or not to expand a branch.

The Information Gain Ratio of an attribute variable $\mathrm{X}$ on a variable class $\mathrm{C}$ can be expressed as:

$$
\operatorname{IGR}(C, X)=\frac{I G(C, X)}{H(X)}
$$

\subsection{Method assessment}

Taking into consideration the indicators used to evaluate the goodness of a classification method in De Oña et al. (2011) and Mujalli and De Oña (2011), and that the variable class used shows 2 possible response categories (state A and state B), the parameters that can be defined are described below:

- Accuracy - The method's precision, defined as the percentage of cases correctly classified by the classifier.

- Sensitivity - The proportion of cases correctly classified as state A among all the observed as state A.

- Specificity - The proportion of cases correctly classified as state B among all the observed as state B.

- Receiver Operating Characteristic Curve (ROC) Area - This indicator represents the curve of positive cases correctly classified (sensitivity), as opposed to the cases of false positives (1-specificity), in such a way that a value 1 describes a perfect adjustment.

If the variable class is accident severity and its potential states are accidents with slightly injured -SI- (state A) and accidents with killed or seriously injured -KSI- (state B), the equations that define these indicators are:

$$
\text { Accuracy }=\frac{T S I+T K S I}{T S I+T K S I+F S I+F K S I} 100 \%
$$




$$
\begin{aligned}
& \text { Sensitivity }=\frac{T S I}{T S I+F K S I} 100 \% \\
& \text { Specificity }=\frac{T K S I}{T K S I+F S I} 100 \%
\end{aligned}
$$

Where, TSI - Number of cases of SI; TKSI- Number of cases of KSI; FSI- Number of false cases of SI (i.e. incorrectly classified as SI); FKSI- Number of false cases of KSI (i.e. incorrectly classified as KSI).

The software used to build the DTs was Weka (Witten and Frank, 2005), which is an open source freeware, available at: http://www.cs.waikato.ac.nz/ml/weka/

Moreover, in order to obtain a more reliable result for each method (CART, ID3 and C4.5) in classification, a repeated Cross Validation procedure (CV) was used. In our case, we use a 10x10-fold CV. In general, a k-fold CV uses the whole data set, and randomly divides the sample used in the training phase into k sets: Sequentially, each subset is kept to be used as a testing set against the tree model generated by the remaining $\mathrm{k}-1$ subsets. Thus, different $\mathrm{k}$ models are obtained, in which the accuracy of the classifications in the training set (k-1) and in the testing subsets $(\mathrm{k})$ can be evaluated and the optimal tree can be selected.

Finally, a corrected paired t-test implemented in Weka, which is a corrected version of the standard paired t-test, was used to compare the results of the trees generated with the different algorithms. This test checks whether a method is better or worse than another, on average, in all the training and testing data sets based on an initial data set. In our case, we used the classification results from the 100 test set for this test, i.e. the sets obtained from a 10x10-fold $\mathrm{CV}$ procedure. The level of significance used for this paired t-test was 0.1.

It should be pointed out that the ID3 algorithm implemented in Weka allows instances without classification. To compare results, we implemented a similar procedure but classified all the instances of the test set as in Abellán and Masegosa (2010). In the case of no classification, we took into account the decision in the parent node. For sake of simplicity, we call this procedure ID3 too.

\subsection{Rules extraction and validation}

The DT's structure was transformed into rules in order to extract its potentially useful information. The rules make a logic conditional structure of the type " $X \rightarrow Y$ ", where in our case, $\mathrm{X}$ is a set of statuses of several attribute variables; and $\mathrm{Y}$ is only one state of the class variable:

IF (a set of statuses of several attribute variables) - THEN (status of the class variable).

For example:

IF (accident type=rollover \& atmospheric condition=light rain) THEN (severity=slightly injured accident).

The part $\mathrm{X}$ of the rule is called the antecedent and the part $\mathrm{Y}$ is called the consequent.

In a DT, rules are configured from the root node, which is where the conditioned structure (IF) begins. Each variable that intervenes in tree division makes an IF of the rule, which ends in child nodes with a value of THEN, which is associated with the state resulting from the child node. The resulting state is the status of the class variable that shows the highest number of cases in the child node analyzed.

A priori, as same number of rules can be identified as the number of terminal nodes on the tree. However, 3 parameters were used on each possible rule " $\mathrm{X} \rightarrow \mathrm{Y}$ ", in order to extract significant 
rules that could provide useful information for the implementation of road safety strategies in the future.

It is known as support of $X$, as the percentage of the data set where appears $X$. In the same vein, we can talk about the support of the entire rule, as the percentage of the data set where $\mathrm{X} \& \mathrm{Y}$ appear. For each rule, the 3 parameters that we use here are the following: Support (S), which for us will be the support of the entire rule; Population (Po), which is the support of the antecedent of the rule; and Probability $(\mathrm{P})$, which is the percentage of cases in which the rule is accurate (i.e. $\mathrm{P}=\mathrm{S} / \mathrm{Po}$ expressed as percentage).

Probability parameter $(\mathrm{P})$ is equivalent to the confidence parameter used in Montella et al. (2011). Montella et al. (2011) set a minimum value $\geq 1 \%$ due to the nature of the data, significant interest in fatal crashes and sample size. However in our paper, the sample size is smaller and the sample is balanced. Toto ensure that the patterns identified by the rules could be extrapolated, the threshold values were: $\mathrm{P} \geq 60 \%$. Other works, including Paned and Abdel-At (2009) established this value at $10 \%$. Based on the sample size, a minimum value (1\%) was also indicated to ensure that the patterns identified by the rules would be representative.

We used the most widely-used parameters in the literature on association rules ( $\mathrm{Po}$ and $\mathrm{P}$ ) and the lowest values, to ensure that the selected rules would be representative: $\mathrm{Po} \geq 1 \%$ and $\mathrm{P} \geq 60 \%$. With these values and due the relation among 3 parameters, $S$ must verify that actually $S \geq 0.6 \%$, a higher value than the one used in Montella et al. (2011), $\mathrm{S} \geq 0.1 \%$, due to reasons related to the special data set they used. It is worth highlighting that if other minimum values were established, more rules could be obtained.

In order to tested that spurious rules, and due to the large number of patterns considered, DTs could suffer from an extreme risk of type-1 error, that is, of finding patterns that appear due to chance alone to satisfy constraints on the sample data (Webb, 2007). To reduce this error and following other authors (Montella et al., 2011; Kashani and Mohaymany, 2011), the dataset was split randomly in two parts: a training set (70\% of the data) and a testing set (remaining 30\%).

The training set was used to build a DT and obtain the significant rules that satisfied the three parameters defined (S, Po, and P). Next, the rules were validated in the testing set to prevent spurious rules (checking that they still met minimum values $\mathrm{S}, \mathrm{Po}$ and $\mathrm{P}$ ).

We also used a binomial test to check if the rule support measure deviates significantly (at 0.05 level) from the theoretically expected value (values from the training set) when the antecedent and the consequent items are independent.

Also, we have used a binomial test to check if the deviations of the rule support measure are significant (at 0.05 level) from the theoretically expected value (values from the training set) when the antecedent and the consequent items are independent.

\subsection{Importance of the variables}

The importance of the variables that intervene in the model is defined for a variable $\mathrm{X}$ with possible states $\left\{\mathrm{x}_{1} \ldots \mathrm{x}\right\}$ by the following equation:

$$
\text { VIM } X=\sum_{i=1}^{h} \frac{n x_{i}}{n}\left(I\left(C / X=x_{i}\right)-I(C)\right)
$$

Where $C$ is class variable (severity), $n_{x i}$ the number of cases that $X=x_{i}, n$ the number of total cases. I is Gini Index in CART, Information Gain in ID3 and Information Gain Ratio in C4.5

\subsection{Description of the data}


Accident data were obtained from the Spanish General Traffic Accident Directorate (DGT) for rural highways in the province of Granada (South of Spain) over a period of 7 years (20032009). In this study, rural highways with only two lanes (one for each direction) were used. The horizontal curves radius of these roads ranged from $16 \mathrm{~m}$ to $2,824 \mathrm{~m}$. And the AADT ranged from 210 to $8,681 \mathrm{veh} /$ day. The accidents analyzed involved 1 vehicle and they did not occur on intersections. The total number of 1,801 accidents met these conditions

Following previous studies (Chang and Wang, 2006; De Oña et al, 2011; Kashani and Mohaymany, 2011), severity of accident was defined according to the worst injured occupant, and two level of severity were identified: accident with slightly injured (SI) and accidents with killed or seriously injured (KSI).

To identify the main factors that affect accident severity, 19 variables were analyzed (see Table

1). The variables chosen were based on:

- Variables available in the original dataset (from DGT).

- Variables selected in others studies with similar objectives (Chang and Wang, 2006; De Oña et al., 2011; Kashani and Mohaymany, 2011; Pakgohar et al., 2010).

The variables describe characteristics related to the driver (age and gender); accident (month, time, day, number of injuries, occupants involved, accident type and cause); road (pavement width, lane width, shoulder type, paved shoulder, pavements markings and sight distance); and context (atmospherics factors and lighting). Some variables were recoded in a reduced number of categories to able to work with them. For instance, in the original dataset MON had 12 categories (12 months), and it was recoded into four periods (see Table 1). Other variables, such as CAU, DAY, LAW, LIG, PAS, PAW, ROM, SEX, SHT, SID, were used as they were in the original dataset. Table 1 gives a description of the variables used for the analysis, together with the frequency distribution.

\section{(insert Table 1 here)}

\section{Results and discussion}

The first step was to build DTs using the three algorithms (CART, C4.5 and ID3) with the aim of classification using 10x10-fold CV procedure. In order to compare the results, corrected paired t-tests were conducted. The results of the tests, comparing the methods to each other on the indicators accuracy, sensitivity, specificity and ROC_Area are shown in Table 2.

\section{(insert Table 2 here)}

C4.5 and CART show similar values for accuracy. ID3 shows significantly worse values than the other two algorithms. The accuracy values are within the range of values obtained in other studies in which classification methods with similar objectives were applied: Abdel Wahab and Abdel-Aty (2001) obtained 61\% accuracy when they applied Bayesian networks and $58.1 \%$ accuracy on neural networks. De Oña et al. (2011) obtained 58\%, 59\% and 61\% accuracy applying Bayesian networks with different algorithms (AIC, MDL and BDeu, respectively).

The C4.5 algorithm gives a higher value than CART (55\% vs. 54\%) in the sensitivity parameter analysis. The improvement is not significant, however. CART gives a higher value than C4.5 for the specificity parameter, although the improvement is not significant either. For ID3, both sensitivity and specificity are poorer, in comparison to the values of the other two algorithms. A global measure given by the ROC Area indicator shows that CART gives the best results $(57 \%)$ whereas ID3 obtains the lowest values again (53\%).

The computational time it took each algorithm to build the DT was another indicator analyzed. It was obtained that the CART method requires the most time to build a tree, being 55 times 
slower than for the $\mathrm{C} 4.5$ algorithm and 42 times slower than ID3. C4.5 is the algorithm that takes the less time, needing only 0.03 seconds to build a DT with 19 variables and 1,801 data. This result is logical because the CART algorithm is more complex, and in turn, C4.5 is more complex than ID3, since it has more optimization parameters in order to improve the results. The implementation of the C4.5 algorithm is optimized in Weka, and therefore the computational time is lower than for ID3.

Taking the above results in consideration, it can be seen that the ID3 algorithm is the method that gives the worst results. The difference in improvement using CART and C4.5 is not significant, however. Although CART obtains slightly higher values in the precision and specificity parameters analyzed, the improvement is not significant, and therefore, we cannot assert a priori that one method is better than the other. It would be worthwhile to analyze the decision rules obtained with the algorithms that attained the best results: C4.5 and CART.

\subsection{CART}

Figure 1 shows the DT built using the CART method with $70 \%$ of the data for training and the remaining data (30\%) for testing, as used by Montella et al. (2011). The CART method creates a tree with 19 nodes and 10 terminal nodes.

\section{(insert Figure 1 here)}

Table 3 shows a description of the six rules identified in the DT that verify the minimum values of the parameters S, Po and P in the training and in the test sets. Support varies from $1.6 \%$ (rule 16) to $8.0 \%$ (rule 6 ). All the rules include at least $1 \%$ of the population, and probability values are higher than $60.9 \%$, with $70.7 \%$ being the highest value (rule 15 ).

With regards to the binomial test that was performed, all the rules obtained from the training set with the minimum threshold have a grade of lift (see Montella et al., 2011) different than 1. Hence the antecedent and consequent are independent. These results were not included in the paper because they are not important for our aims. The binomial test showed that all the rules given in Table 3 have no significant differences (at 0.05 level), based on support when they are applied on the test set. Only the rule 5 (see Table 3 ) has a high level of support in the test set compared to the support in the training set. This difference is significant at 0.05 level of significance.

\section{(insert Table 3 here)}

The root variable that generates the tree is SEX (see Figure 1) which splits into two branches (nodes 1 and 2). For female drivers, and depending on LIG, nodes 5 and 6 are obtained, with different degrees of severity (see Figure 1): accidents are KSI if LIG is insufficient or without lighting, with a probability of $61 \%$ (rule 5); while if LIG is sufficient, dusk or day light the severity is SI, with a probability of $69 \%$ (rule 6).

The rest of the rules are attributable to male drivers (node 1). After this node, the tree splits according to ACT. For rollover, collision with obstacles and other accidents types, the severity of the accident is SI, with this rule representing 6.4\% of the population (rule 4 in Table 3). For all other accident types, the tree splits by the variable ATF: if ATF is light rain the accident is SI, with a probability of $63 \%$ (rule 8); in others cases the tree continues to grow according to DAY. If DAY is public holiday or after public holiday the accident is KSI, with a probability of 65\% (node 10 in Figure 1), and if it is before public holiday or working day the tree is divided according to TIM. From 6-12 h, accidents with SI are obtained when PAS is paved or nonexistent (rule 15, which is the one that represents the highest probability: almost $71 \%$ ) whereas when it is not paved the severity is KSI (rule 16). From 12-6 h, tree is divided by MON and LIG (see Figure 1), however neither of the obtained nodes are rules. 
Following Eq. 8, it is possible to obtain the importance of the variables in the model. Table 4 shows normalized importance of these variables. 12 variables were detected as having the greatest influence on accident severity, with percent which varying from $100 \%$ to $9.9 \%$.

\section{(insert Table 4 here)}

LIG is the most important variable, coinciding with previous studies. Gray et al. (2008) identified that more severe injuries are predicted during darkness. Abel-Aty (2003) and Heali et al. (2008) found the same results. Pande and Abel-Aty (2009) concluded that there is a significant correlation between lack of illumination and high severity of crashes. De Oña et al. (2010) also pointed that KSI accidents are associated with roadways without lighting.

ATF is the second variable with $83.6 \%$ importance in the model. This result matches with other previous studies, such as Xie et al. (2009) and Mujalli and De Oña (2011). TIM has 77.1\% importance in the model which is coherent because there is already a degree of relationship between the time and lighting variables. Next comes ACT with 76.0\% (Kcoleman and Kweon, 2002, De Oña et al., 2011 also found this variable as one of the most important in the study of severity). SEX represented $72 \%$ of the variables' importance. The other variables (see Table 4) in the model are less important, with percentages between $55.9 \%$ and $9.9 \%$.

\subsection{C4.5}

Figure 2 represents a DT built using the C4.5 algorithm based on the training set. It shows 52 nodes, with 39 terminal nodes. The increase in the number of nodes is justified be the fact that this algorithm creates a branch for each category of variable used in the analysis. In this case, however, only 9 rules that meet the minimal values for support, population and probability were obtained (see Table 5).

\section{(insert Figure 2 here)}

Since the tree generated with C4.5 is larger, only the rules extracted in Table 5 are used to describe the following tree structure. In this case, the rules in Table 5 also verify the minimum values of the parameters S, Po and P in the training and in the test sets. For C4.5, the binomial test showed that all the rules obtained from the training set with the minimum threshold have a grade of lift (see Montella et al., 2011) different than 1. And none of the rules given in Table 5 have significant differences (at 0.05 level) on support when they are applied to the test set.

\section{(insert Table 5 here)}

As in CART, the root variable is the variable SEX. For female drivers when LIG is daylight, the rule with the highest population (9.9\%) and support (6.8\%) gives a severity result of SI (rule 6 in Table 5).

Most of the tree is generated by male drivers (see Figure 2) and according to ACT. Figure 2 and Table 5 show the following patterns: if ACT is rollover the severity is SI, with a probability of $61 \%$ (rule 12 in Table 5); whereas, if ACT is collision with pedestrian and PAS is paved the severity is KSI. This is the rule with the highest probability (78\%). The rest of the rules are obtained for rollover accidents and depending on CAU.

When CAU is a combination of factors, SID is without restriction and MON is spring the severity of accident is SI with almost $74 \%$ of the probability (rule 33). For CAU attributable to driver and depending on VEH the following patterns are shown: when VEH is a truck the accident is $\mathrm{KSI}(67.5 \%)$, rule 29; when is a motorbike or motorcycle and PAS is non existent or 
impassable, the same severity (KSI) is obtained (rule 40); and for car two more rules are obtained depending on PAW.

When PAW is between [7-6] meters and driver's age is $27-60$, rule 46 is obtained, the severity is SI with a probability of almost $68 \%$. When PAW is $>7$ meters, the tree splits according to NOI, and when it is higher than 1, accidents are SI in $64.4 \%$ of cases (rule 48 in Table 5).

Following Eq. 8, it is possible to obtain the importance of the variables in the C4.5 model (see Table 6).

\section{(insert Table 6 here)}

Fourteen variables were detected as having the greatest influence on accident severity, with percent which varying from $100 \%$ to $11.2 \%$. ACT is the most important variable in the C4.5 model, followed by CAU. These results are in accordance with Al-Ghamdi (2002) and Kashani and Mohyamany (2011), who situate crash cause among the top variables influencing severity. The CART algorithm identified eleven of the previous fourteen variables. Moreover C4.5 identified VEH, PAW, and NOI.

\section{Conclusions}

DTs allow accident classification based on crash severity. They provide an alternative to parametric models due to their ability to identify patterns based on data, without the need to establish a functional relationship between variables. Moreover, such classification models can be used to determine interactions between variables that would be impossible to establish directly, using ordinary statistical modeling techniques.

The main conclusions regarding the methods used in this paper to build DTs are the following:

- CART builds binary DTs and therefore certain categories of splitting variables are grouped in some branches, increasing node support, but making it impossible to analyze the influence of a specific category on severity. $\mathrm{C} 4.5$ creates a branch for each category, thereby permitting an analysis of the influence of all the categories of variables used to build the DT. Consequently, it could be said that the rules obtained with CART are less informative.

- C4.5 generates DTs with more branches than CART, and therefore it produces more rules. However, not all the rules meet the established minimal number of support, population and probability parameters, and therefore the rules may not be very useful for implementing future road safety strategies.

- The importance of the variables in the model can be obtained using either algorithm.

- The two algorithms have certain similarities with regards to the structure of the tree generated. For example, the root variable for both is SEX and tree density is obtained by the branch male drivers, and the value that continues to split the tree is ACT.

DTs permit certain potentially useful rules to be determined that can be used by road safety analysts and managers. Initially, they should focus on severe crashes and subsequently intervene in minor accidents. The approach proposed in this paper within each group will enable actions to be prioritized on the basis of support, population and probability. It is worth highlighting certain overall conclusions from a road safety perspective.

The rules drawn from the two methods are coincidental in that:

- Male drivers are the main causes of KSI crashes.

- The probability of KSI increases if pedestrians are involved (node 3 Figure 1 and rule 11 in Table 5).

- When women drivers are involved in an accident, both methods predict SI when lighting exists (full daylight, sufficient lighting and dusk) (rule 6 in Tables 3 and 5, and nodes 2 and 3 in Figure 2). However, both methods predict KSI when the lighting is 
non-existent or insufficient (rule 5 in Table 3 and nodes 6 and 7 in Figure 2). These rules are not observed for men and may indicate that women increase their risk of severity under conditions of less lighting on the road.

From the standpoint of road safety, most of the rules extracted coincide with the conventional problems found on rural highways in developed countries, as most previous studies point out. This validates the method proposed in this paper, and therefore it is positive. However, the primary importance of this proposal is that other data bases not used here (i.e. other infrastructure, roads and countries) could be used to identify unconventional problems in a manner easy for road safety managers to understand, as decision rules.

However, using these two types of DTs permitted the identification of a specific problem worthy of further study: Although less women than men are involved in accidents $(15.3 \%$ vs. $84.5 \%$, see Table 1), and accident severity is SI in $62.2 \%$ of cases, the two methods indicate that women increase their risk of severity under conditions of non-existent or insufficient lighting. The efforts of multidisciplinary teams with experts on psychology, physiology, road safety and illumination should focus on a search of the reason why women, contrary to men, present higher risk of severity under conditions of less lighting on the road.

Finally, it should be stressed that each method has advantages and drawbacks, and reveals different information. Therefore, the two methods complement each other and the recommendation is to use both of them for a full analysis.

\section{Future work}

When we use a DT to obtain decision rules, such rules are highly dependent on the variable entered in the root node, which permits knowledge to be extracted only in the sense dictated by said root variable. For future research, it is worth studying the possibility of generating DTs by varying the root node and analyzing all the rules that may be obtained from a single set of data.

For the same purpose, we would like to apply new split criteria based on new mathematical models for representing information, as well as the new procedures used in classification to date. These criteria and procedures can be seen in Abellán and Masegosa (2010) and Abellán et al. (2011).

\section{Acknowledgements}

The authors express their gratitude to the Spanish General Directorate of Traffic (DGT) for supporting this research and offering all the resources that are available to them. Griselda López wishes to express her acknowledgement to the regional ministry of Economy, Innovation and Science of the regional government of Andalusia (Spain) for their scholarship to train teachers and researchers in Deficit Areas, which has made this work possible.

\section{REFERENCES}

Abdel Wahab, H.T., Abdel-Aty, M.A., 2001. Development of artificial neural network models to predict driver injury severity in traffic accidents at signalized intersections. Transportation Research Record 1746, 6-13.

Abdel-Aty, M., 2003. Analysis of driver injury severity levels at multiple locations using ordered probit models. Journal of Safety Research 34, 597-603.

Abellán, J., Baker, R.M., Coolen, F.P.A., 2011. Maximising entropy on the nonparametric predictive inference model for multinomial data. European Journal of Operational Research 212(1), 112-122.

Abellán, J., Masegosa, A., 2010. An ensemble method using credal decision trees. European Journal of Operational Research, 205(1), 218-226.

Agrawal, R., Imielinski, T., Swami, A., 1993. Mining association rules between sets ofitems in large databases. In: Proceedings of ACM SIGMOD Conference on Management of Data (SIGMOD 1993), 207-216. 
Al-Ghamdi, A., 2002. Using logistic regression to estimate the influence of accident factors on accident severity. Accident Analysis and Prevention 34 (6), 729-741.

Breiman, L., Friedman, J.,Olshen, R., and Stone, C., 1984. Classification and Regression Trees. Belmont, CA: Chapman \& Hall.

Chang, L.Y., Wang, H.W., 2006. Analysis of traffic injury severity: an application of nonparametric classification tree techniques. Accident Analysis and Prevention 38, 1019-1027.

De Oña, J., Mujalli, R.O., Calvo, F.J., 2011. Analysis of traffic accident injury on Spanish rural highways using Bayesian networks. Accident Analysis and Prevention 43, 402-411.

Fayyad, U. M., Piatetsky-Shapiro, G., Smyth, P., 1996. From Data Mining to Knowledge Discovery: An Overview. In: Fayyad, U.M., Piatetsky-Shapiro, G., Smyth, P., Uthurusamy, R. (Eds.), Advances in Knowledge Discovery and Data Mining. AAAI Press/The MIT Press, 1-34. Gray, R.C., Quddus, M.A., Evans, A., 2008. Injury severity analysis of accidents involving young male drivers in Great Britain. Journal of Safety Research 39, 483-495.

Kashani, A., Mohaymany, A., 2011. Analysis of the traffic injury severity on two-lane, two-way rural roads based on classification tree models. Safety Science 49, 1314-1320.

Kashani, A., Mohaymany, A., Ranjbari, A., 2011. A Data Mining Approach to Identify Key Factors of Traffic Injury Severity. Promet-Traffic \& Transportation, 23 (1), 11-17.

Kuhnert, P.M., Do, K.A., McClure, R., 2000. Combining non-parametric models with logistic regression: an application to motor vehicle injury data. Computational Statistics \& Data Analysis, Vol 34(3), 371-386.

Liu, P., 2009. A self-organizing feature maps and data mining based decision support system for liability authentications of traffic crashes. Neurocomputing 72, 2902-2908.

Montella, A., Aria, M., D’Ambrosio, A.,Mauriello, F., 2011. Analysis of powered two-wheeler crashes in Italy by classification trees and rules discovery. Accident Analysis and Prevention, in press.

Mujalli, R.O., De Oña, J. (in press). Injury Severity Models for Motorized Vehicle Accidents: A review, Proceedings of the Institution of Civil Engineering - Transport. In Press.

Mujalli, R.O., De Oña, J., 2011. A method for simplifying the analysis of traffic accidents injury severity on two-lane highways using Bayesian networks. Journal of Safety Research, 42, 317326

Pakgohar, A., Tabrizi, R.S., Khalilli, M., Esmaeili, A., 2010. The role of human factor in incidence and severity of road crashes based on the CART and LR regression: a data mining approach. Procedia Computer Science 3, 764-769.

Pande, A., Abdel-Aty, M., 2009. Market basket analysis of crash data from large jurisdictions and its potential as a decision supporting tool. Safety Science 47, 145-154.

Quinlan, J. R. 1993. C4.5: Programs for Machine Learning. Morgan Kaufmann Publishers, San Mateo, California.

Quinlan, J.R., 1986. Induction of decision trees. Machine Learning, 1(1), 81-106.

Savolainen, P., Mannering, F., Lord, D., Quddus, M., 2011. The statistical analysis of highway crash-injury severities: A review and assessment of methodological alternatives. Accident Analysis and Prevention. In press

Shannon, C., Weaver, W., 1964. The Mathematical Theory of Communication. The University of Illinois.

Sohn, S.Y., Shin, H.W., 2001. Data mining for road traffic accident type classification. Ergonomics 44, 107-117.

WHO, World Health Organisation, 2009. Informe Global sobre el estado de la Seguridad Vial: Tiempo para la Acción. Available at:

www.who.int/violence_injury_prevention/road_safety_status/2009

Webb, G.I., 2007. Discovering significant patterns. Machine Learning 68, 1-33.

Xie, Y., Zhang, Y., Liang, F., 2009. Crash Injury Severity Analysis Using Bayesian Ordered Probit Models. Journal of Transportation Engineering ASCE, 135(1), 18-25. 


\section{List of figures:}

Figure 1. Decision tree built with CART.

Figure 2. Decision tree built with C4.5. 


\section{List of tables:}

Table 1. Variables used from the police accident reports.

Table 2. Comparison of the parameters produced by the various algorithms.

Table 3. Description of the rules according to the CART.

Table 4. Importance of the variables with CART.

Table 5. Description of the rules according to the C4.5.

Table 6. Importance of the variables with C4.5. 